MATEC Web of Conferences 13,04001 (2014)

DOI: $10.1051 /$ matecconf/ 20141304001

(C) Owned by the authors, published by EDP Sciences, 2014

\title{
Morphology, Tensile Strength and Oil Resistance of Gum Rubber Sheets Prepared from Lignin Modified Natural Rubber
}

\author{
Asrul M. ${ }^{{ }^{*}}$, Othman M. ${ }^{2}$, Zakaria M. ${ }^{3}$, and Ahmad-Nazir K. ${ }^{4}$ \\ ${ }^{1}$ Chemistry and Materials Exploratory Unit, MRB Experimental Station, $47000 \mathrm{Sg}$ Buloh, Selangor, \\ Malaysia \\ ${ }^{2}$ Department of Mechanical Engineering, Universiti Teknologi PETRONAS, Bandar Seri Iskandar, \\ 31750 Tronoh, Perak, Malaysia \\ ${ }^{3}$ Department of Chemical Engineering, Universiti Teknologi PETRONAS, Bandar Seri Iskandar, \\ 31750 Tronoh, Perak, Malaysia \\ ${ }^{4}$ Tyre Testing Laboratory, MRB Experimental Station, $47000 \mathrm{Sg}$ Buloh, Selangor, Malaysia. \\ ${ }^{*}$ Corresponding author: asrul@1gm.gov.my
}

\begin{abstract}
The paper describes the preparation of lignin filled natural rubber latex composite and its subsequent use to obtain lignin modified rubber. Two types of lignin i.e.: rubber wood and commercial alkali lignin were used as rubber filler. Gum rubber sheets were prepared from the lignin modified rubber and their properties were compared to Standard Malaysian Rubber (SMR 20) and a type of rubber obtained from the coagulation of high ammonia latex. Rubber morphology was investigated using Scanning Electron Microscope on the cross-sectional area of cryo-fractured samples. Oil resistance of the rubber sheets was determined by measuring the mass change before and after ASTM IRM 903 oil immersion, while the tensile strengths were determined according to ASTM D412 standard. Low values of tensile strength obtained for the commercial alkali lignin modified rubber sheet relative to the rest of the rubber samples was attributed to poor lignin dispersion. This occurrence was substantiated by the SEM analysis of cryofractured samples where crazes and inhomogeneity was observed. Nonetheless, both lignin modified rubbers exhibited higher level of oil resistance compared to SMR 20. This is due to the nature of lignin as a hydrophilic component and its presence in the rubber matrix complicates the oil diffusion process into rubber.
\end{abstract}

\section{Introduction}

Lignin is a complex polymer which is one the one of the major component in the structure of plants. In terms of material sustainability, lignin can be considered as a renewable raw material or sustainable chemical compound. The utilization of lignin in rubber can be traced back to the post World War era with the purpose of replacing carbon black as rubber filler after the $2^{\text {nd }}$ World War. This research approach was encompassed in the US Government Synthetic Rubber Program [1] in 1941 due to the scarcity of rubber and other raw materials during the World War. In addition, the utilisation of lignin was also motivated by its potential availability from the annual production of lignin amounting to more than a million tons from the pulp mills in the United States in the post war era. One of the works

This is an Open Access article distributed under the terms of the Creative Commons Attribution License 2.0, which permits unrestricted use, distribution, and reproduction in any medium, provided the original work is properly cited. 
at the time, as described by Keilen and Pollak [2] was the use of lignin derived from the black liquor of pulp sulphate mill as filler in synthetic rubber i.e.: styrene butadiene rubber. Their findings revealed that the tensile strength of lignin filled rubber to be similar to carbon black filled vulcanised rubber. The other advantage was the rubber containing lignin had lower specific gravity compared to the carbon black filled rubber which rendered the rubber composite lighter in weight.

Most of the work with regards to lignin utilisation rubber involves mixing of lignin in rubber latex to obtain lignin filled rubber latex composite which is then coagulated to form a lignin masterbatch for subsequent use as an ingredient in rubber compounding. For instances, Tibenham and Grace [3] worked on the partial replacement of carbon black with lignin and showed an improvement of 13.8\% in tensile strength. In another study, Sagajllo [4] showed that various HAF black and lignin combinations in rubber exhibited higher tensile strength with marked improvements of up to $20 \mathrm{MPa}$ can be obtained if the lignin was prepared via lignin masterbatch technique. Thus, the utilisation of lignin as a masterbatch in rubber compounding is not a novel approach and it can be regarded as the conventional method of lignin incorporation in rubber.

In our previous work [5], we used sulphur free lignin derived from rubber wood as filler in natural rubber latex to enhance the oil resistance properties and tensile strength of the resulting latex films. We concluded that at $10 \mathrm{wt} \%$ lignin loading, the increase of $60 \%$ tensile strength observed was contributed by the adequate dispersion level of lignin dispersion and higher level of chain entanglement as indicated by Transmission Electron Microscopy (TEM) analysis and elastic constant $\left(\mathrm{C}_{2}\right)$ measurements respectively. The increment of oil resistance as measured by mass change in ASTM IRM 903 oil immersion was increased by 6\%. Therefore, it is shown that the level of lignin loading at $10 \mathrm{wt} \%$ was sufficient to impart certain level of improvements in the properties of rubber films.

Hence we believed that similar improvements in gum rubber sheets can be achieved using the lignin masterbatch technique to prepare lignin modified rubber which shall be used further as a raw rubber to simplify the rubber processing for the utilisation of lignin modified rubber. In our present investigation, we propose the use of lignin modified rubber with $10 \mathrm{wt} \%$ lignin loading from coagulated lignin filled rubber latex composite. The lignin modified rubber obtained after coagulation is directly used as a raw rubber to produce gum rubber sheets. The changes on the rubber morphology influenced by the presence of lignin were examined using SEM cryo-fracture. The tensile strength and oil resistance were also compared to Standard Malaysian Rubber (SMR 20) and a control rubber obtained from the high ammonia (HA) rubber latex coagulation where both were devoid of lignin.

\section{Experimental}

\subsection{Materials}

In the present study, two types of lignin are used. A type of commercial alkali lignin (AL) containing $3.64 \%$ sulphur was purchased from Sigma-Aldrich (Sigma-Aldrich, USA). Another type of lignin, rubber wood (RW) lignin used was derived from rubber wood RRIM Clone Series 2000 by means of alkaline digestion at $150{ }^{\circ} \mathrm{C}$ and pressure of 4 bars for 30 minutes. Crude lignin in the digestion liquor was further precipitated and isolated by centrifugation at $15000 \mathrm{~g}$. The precipitated lignin was further processed by crushing in liquid nitrogen to acquire a particle size which was compatible with natural rubber latex in the region of 0.05 to $5 \mu \mathrm{m}$. Details of the lignin derivation from rubber wood are described elsewhere [6]. Rubber latex which was used as the base rubber to mix lignin was high ammonia latex (HA) purchased from Lee Rubber (60\% rubber solid content, Malaysia). 


\subsection{Methods}

\subsubsection{Preparation of lignin modified rubber and gum rubber sheet}

For this purpose, RW lignin powder at $10 \mathrm{wt} \%$ loading per dry rubber was added directly into commercial grade high ammonia natural rubber latex and diluted with distilled water to a solid content of $30 \%$. The mixture was then stirred in a baffled circular vessel using a $45^{\circ}$ pitched blade impeller at $200 \mathrm{rpm}$. Subsequently, the lignin filled rubber latex composite was coagulated with $3 \%$ formic acid. The coagulated rubber was then creped using a creper mill and dried in an oven at $70{ }^{\circ} \mathrm{C}$ until dry. Similar method was used to prepare AL lignin modified rubber. A rubber obtained by coagulating HA rubber latex only without the addition of lignin was also prepared which serves as a negative control.

The lignin modified rubber was subsequently used as a raw rubber in rubber compounding following conventional sulphur vulcanisation [7]. Compounding was carried out on a two roll mill and further cured under a hot press. After curing, thin sheets of gum rubber with thickness of approximately $4 \mathrm{~mm}$ were obtained. A commercially available technically specified standard dry rubber used for comparison purpose was Standard Malaysian Rubber (SMR 20, Malaysian Rubber Board).

\subsubsection{Testing}

For Scanning Electron Microscope (SEM) cryo fracture, the test portion of the gum rubber sheet was cut to a small piece and dipped into liquid nitrogen for 30 minutes and fractured while it is still brittle with steel pliers. Specimen was then prepared for examination by evaporative coating with ultra-thin layer of platinum under high vacuum. SEM examinations using a JOEL JSM-6701F operated at 2kV with $15 \mathrm{~mm}$ working distance. Tensile strength measurements were carried out as described in the ASTM D 412 standard [8]. Determination of changes in mass after oil immersion (Oil resistance) was conducted in accordance to ASTM D471 standard [9]. In order to obtain percentages of mass change, the mass differences of the samples after and before immersion were calculated using Equation 1. Whereby, $M_{1}$ is the mass of samples before swelling and $M_{2}$ is the mass of the samples after oil immersion.

$$
\text { Change in mass, } \Delta M, \%=\frac{\left(M_{2}-M_{1}\right)}{M_{1}} \times 100
$$

\section{Results and discussion}

\subsection{Morphology from SEM cryo-fracture analysis}

The morphology of cryo-fracture sample examined under SEM is a common method to determine phase structure and compatibility of polymer blends $[10,11,12]$. The occurrence of crazes or cracks represents immiscibility between the polymer blend further corresponded to the mechanical properties of the blends. The cross-section of the cryo-fracture surfaces of the rubber sheets were examined under SEM and the results are shown in Figure 1. 

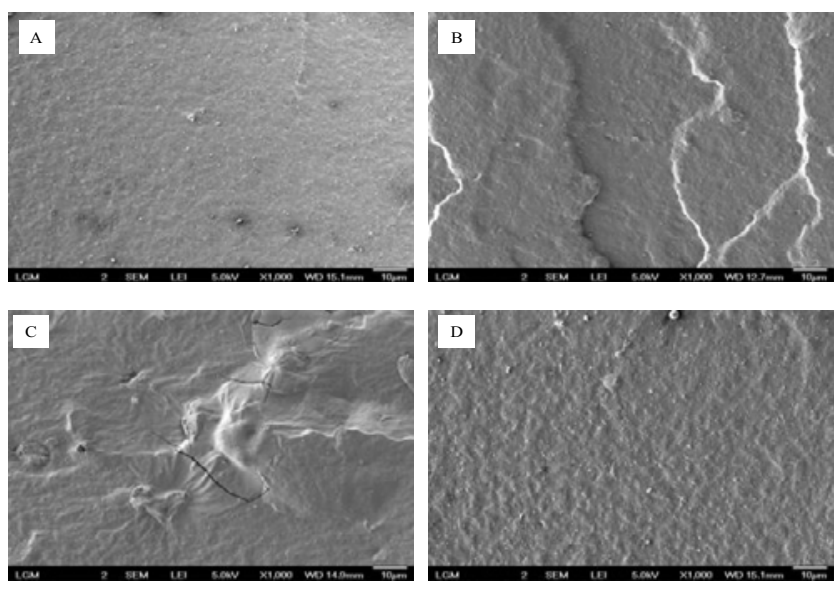

Figure 1. SEM micrographs of cryo-fractured rubber vulcanisates examined at the cross-sectional area. The micrographs A: Standard rubber (SMR 20), B: RW lignin modified rubber, C: AL lignin modified rubber, D: Rubber from coagulated HA latex (negative control).

The morphology of the control (SMR 20) was much similar to the rubber coagulated from HA latex devoid of lignin. It is shown in the micrographs that cryo-fractured surfaces of the control samples exhibited brittle fracture without any heterogeneous phase. While lignin modified rubber, particularly the commercial alkali lignin indicated certain level of heterogeneity. This can be attributed to phase separated structure [13] as observed in hydrophilic/hydrophobic polymer blend. The presence of hydrophilic lignin would induce agglomeration and crazes in the rubber matrix contributing to more stress concentration site [14]. This SEM observation further supported the results of the tensile strength shown in the subsequent section where AL lignin modified rubber sheet exhibited the lowest tensile strength which corresponded to the presence of crazes and cracks.

\subsection{Tensile Strength and Oil resistance}

The purpose of the investigation on oil resistance was to indicate the effect of lignin in improving the oil resistance of natural rubber. The deterioration by oil in rubber was determined by measuring the mass changes before and after immersion in oil. In relation to products performance, certain rubber products such as seal and gaskets are exposed to oils during service, thus this method may be used to simulate service conditions. The oil immersion was conducted to evaluate the comparative ability of the rubber sheets to resist oil swelling and maintains its intactness. The American Society for Testing and Materials (ASTM) IRM 903 oil was used as it has a flash point and aniline point of $163{ }^{\circ} \mathrm{C}$ and $70^{\circ} \mathrm{C}$ respectively, and is considered comparable to typical hydraulic oils [15].

The results of the tensile strengths and oil resistance are shown in Table 1. All the samples tested exhibited tensile strengths above the ASTM stipulated requirements for rubber sheet gasket [16]. The oil resistance as reflected by the percentages of mass change in oil immersion showed that rubber sheets prepared from both RW lignin modified rubber and commercial alkali lignin exhibited better oil resistance relative to the control SMR 20 with smaller changes in mass after immersion. Comparatively, the oil resistance between RW lignin modified rubber and commercial AL alkali lignin was nearly similar. 
Table 1. Tensile strength and oil resistance of rubber sheets

\begin{tabular}{|c|c|c|}
\hline \multirow{2}{*}{ Rubber Samples } & \multicolumn{2}{|c|}{ Properties } \\
\cline { 2 - 3 } & $\begin{array}{c}\text { Tensile Strength } \\
(\mathrm{MPa})\end{array}$ & $\begin{array}{c}\text { Mass change in } \\
\text { oil }(\%)\end{array}$ \\
\hline Standard rubber (SMR 20) & $20.20 \pm 0.30$ & $40.50 \pm 0.84$ \\
\hline RW lignin modified rubber & $21.70 \pm 0.60$ & $36.11 \pm 0.68$ \\
\hline $\begin{array}{c}\text { Commercial AL lignin modified } \\
\text { rubher }\end{array}$ & $14.90 \pm 0.44$ & $35.53 \pm 0.70$ \\
\hline Coagulated HA latex rubber & $19.90 \pm 0.65$ & $39.34 \pm 0.98$ \\
\hline
\end{tabular}

The ability of lignin to resist oil is associated to the hydrophilic lignin structure having substituted phenol with hydroxy and methoxy group which are less soluble in oil. The presence of such hydrophilic component in the rubber matrix complicates the oil diffusion process when concentration gradient as the driving force for oil diffusion as a function of time is no longer constant. The resistance to oil diffusion observed in lignin modified rubber may be attributed the tortuosity introduced by the presence of hydrophilic lignin in the polar rubber matrix may resemble the effect of filler incorporation in composite polymeric materials [17]. A polymer composite such as polymer-clay is usually associated with complex structures which contribute to an increase in path of tortuosity for diffusing molecules to penetrate.

\section{Conclusions}

The SEM cryo-fracture analysis at cross-sectional area exhibited the occurrence of crazes in the AL lignin modified rubber indicative of poor dispersion. Adequate tensile strength and better level of oil resistance is observed in lignin modified rubber relative to the conventional standard rubber (SMR 20). In general, it can be suggested that the use of lignin modified rubber did not alter or changed the rubber morphology tremendously and its usage as rubber sheet gasket with enhancement in oil resistance is viable. RW lignin modified rubber exhibited better tensile strength compared to AL lignin.

\section{References}

1. P. R.Samuelson, Working Paper MIT-EL 76-027W, November, Massachusetts Institute of Technology (1976).

2. J. J. Keilen and Pollak A., Industrial Engineering and Chemistry, 39, 480-483(1947).

3. F.J. Tibenham, and N.S., Grace Industrial Engineering and Chemistry, 46, 824-828 (1954).

4. I.Sagajjlo, Rubber Chemistry and Technology, 30, 639-651 (1957).

5. M. Asrul, M. Othman, M. Zakaria and M. S. Fauzi, Journal of Rubber Research, 16(2), 118-133 (2013).

6. M. Asrul, M. Othman, M. Zakaria and M.S. Fauzi, Malaysian Patent Application, No. PI 2011003945 (2011).

7. Malaysian Rubber Producers' Research Association, The Natural Rubber Formulary and Property Index, pp 220-222. Hertford: Malaysian Rubber Producers' Research Association, (1984). 
8. ASTM Standard D 412-98, West Conshohocken PA, ASTM International (1998).

9. ASTM Standard D 471-06, West Conshohocken PA, ASTM International (2006).

10. F.H. Axtell, P. Phinyocheep and P. Kriengchieocharn, Journal of Science Society of Thailand, , 22, 201-216 (1996).

11. Z. Su, Q. Li, Y. Liu, G-H Hu and C. Wu, European Polymer Journal, , 45, 2428-2433 (2009).

12. H. Liu, F. Chen, B. Liu, G. Estep, and J. Zhang, Macromolecules, , 43, 6058-6066 (2010).

13. M. Kowalczyk, and E. Piorkowska, Journal of Applied Polymer Science, 124, 4579-4589 (2012).

14. J. Wang, W. Wu, W. Wang and J. Zhang, Journal of Polymer Research,, 18, 1023-1032 (2011).

15. J. Hanley, N. Murphy, H. Ali, and S. Jerrams, 11th International Seminars on Elastomers, 23 -27 Sept, Freiburg, Germany (2007).

16. ASTM D1330-04 Standard, West Conshohocken PA, ASTM International (2004).

17. D.D. Kee, Q. Liu, and J. Hinestroza, Canadian Journal of Chemical Engineering, 83, 913-929 (2005). 